\title{
Acne phototherapy with a $1450-n m$ diode laser: an open study
}

\author{
Natusko Konishi \\ Hideki Endo \\ Naoki Oiso \\ Shigeru Kawara \\ Akira Kawada \\ Department of Dermatology, Kinki \\ University School of Medicine, Ohno- \\ Higashi 377-2, Osaka-Sayama city, \\ Osaka 589-85II, Japan
}

\begin{abstract}
The purpose of this study was to investigate the efficacy of phototherapy with a newly-developed 1450-nm diode laser in patients with mild to moderate acne. An open study was performed in acne patients who were treated up to five times with a two week interval. Acne lesions were reduced by $63 \%$. Only one patient discontinued treatment due to vesicle formation as an adverse effect. Phototherapy using this diode laser source was effective and well tolerated in acne patients, suggesting that this phototherapy may be a new modality for the treatment of acne.
\end{abstract}

Keywords: acne vulgaris, phototherapy, 1450-nm diode laser, sebaceous gland

\section{Introduction}

Acne vulgaris is the most common dermatologic disorder. It affects approximately $85 \%$ of individuals aged between 12 and 24 years at some time (Leyden 2003). Various modalities have been performed for acne treatments, eg, topical and oral antibiotics, retinoic acids, and phototherapies (Cunliffe and Goulden 2000; Kawada, Aragane, Tezuka, et al 2002; Van De Kerkhof et al 2006). In phototherapies, blue light sources (Sigurdsson et al 1997; Papageorgiou et al 2000; Kawada, Aragane, Kameyama, et al 2002) and photodynamic therapy (PDT) using topical 5aminolevulinic acid (ALA-PDT) (Hongcharu et al 2000) have been reported to be effective for acne vulgaris. Blue light is a theoretically effective phototherapy since exposure to blue visible light induces photoexcitation of porphyrins from Propionibacterium acnes, singlet oxygen production, and subsequent bacterial destruction (Kawada, Aragane, Kameyama, et al 2002). ALA-PDT utilizes visible light-induced phototoxic reaction of ALA-derived protoporphyrin IX (PpIX) accumulated in the target lesions of acne (Hongcharu et al 2000).

Recent reports demonstrate that laser therapies including 532-nm potassium titanyl phosphate laser, 585-nm pulsed dye laser, 1450-nm diode laser, and 1540-nm Er:glass laser are clinically effective in acne vulgaris (Friedman et al 2004; Mariwalla and Rohrer 2005; Nouri and Ballard 2006). We performed an open study of a newlydeveloped $1450 \mathrm{~nm}$ diode laser as phototherapy of acne vulgaris. This diode laser was shown to be effective and well tolerated by acne patients, suggesting that this diode laser therapy could be a possible new treatment modality for acne.

\section{Patients and methods}

\section{Patients}

This study was an open clinical trial performed at our department from December 2005 to June 2006. Twenty-seven female patients with mild to moderate acne lesions involving the face and/or the back and/or the chest participated in this study. Mild to moderate acne was determined according to the definition by Glass and colleagues 
(1999). Patients had to have between 15 and 100 inflammatory lesions and/or between 15 and 100 noninflammatory lesions and no more than 3 nodules to be included (Glass et al 1999). The average age was 23 years (range 16-34). No medication was administered during the 4 weeks prior to the study. The aims of the study were explained to the patients and informed consent was given.

\section{Treatment protocol}

A 1450-nm diode laser (Smoothbeam ${ }^{\mathrm{TM}}$, Candela Corporation, Wayland, MA, USA) (Figure 1) was used for all treatments. This apparatus with the dynamic cooling device emitted light at $1450 \mathrm{~nm}$ with repetition rate of $1 \mathrm{~Hz}$ and pulse duration of $210 \mathrm{~ms}$. Treatment fluence was 12.5 $\mathrm{J} / \mathrm{cm}^{2}$ with a spot size of $6 \mathrm{~mm}$. Each patient received treatments up to five times with a two week interval; other therapies were prohibited during this time. Both eyes were protected with an eyeglass to prevent unexpected adverse effects of the light during this treatment.
Clinical assessment was performed 5 times during the trial period $(0,2,4,6,8$, and 10 weeks). First, the number of lesions, eg, papules, pustules, cysts, and nodules, was counted. The secondary criterion was the investigator's global improvement rating on a five-point scale (-1, worsened; 0, unchanged; 1, improved; 2, markedly improved; and 3, resolved). Tolerance was assessed by asking patients about any signs or symptoms of adverse reactions.

\section{Results}

\section{Patients}

Twenty-seven female patients were recruited for the study. One patient discontinued treatment after the first treatment due to an adverse effect of vesicle formation. Four patients stopped after the second treatment, 4 patients stopped after the third, and 5 stopped after the fourth. Eleven patients stopped because skin lesions improved, while two stopped for nonmedical reasons after the second treatment. The

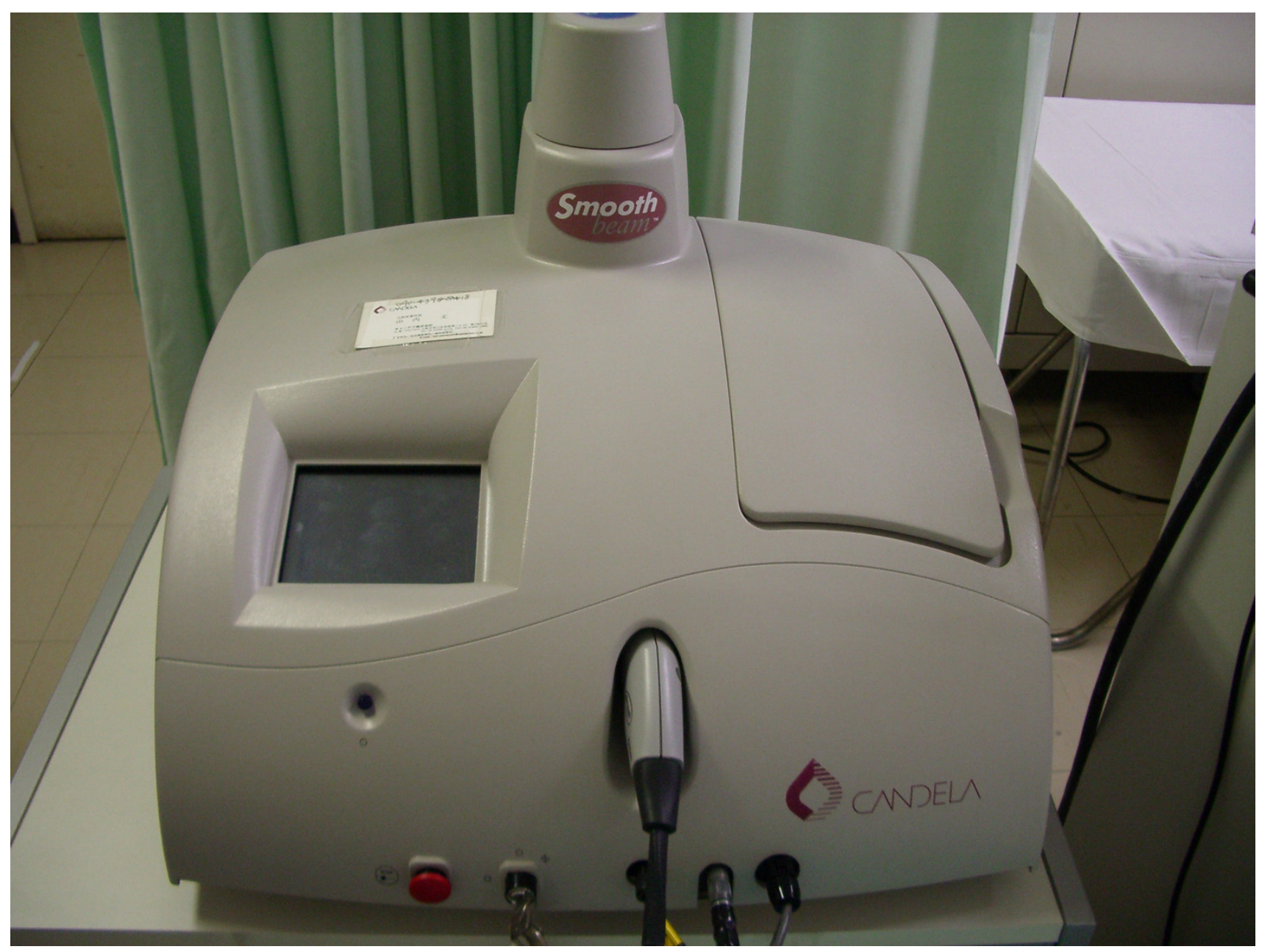

Figure I The apparatus of I450-nm diode laser with a dynamic cooling device. 
numbers of lesions (mean \pm standard deviation [SD]) before the treatment were $23.8 \pm 18.1$.

\section{Clinical efficacy}

The number of lesions before treatment was $23.8 \pm 18.1$ (mean \pm SD) $(n=27)$. The number of lesions were: $20.5 \pm$ 14.1 at 2 weeks after 1 treatment $(n=27) ; 17.1 \pm 13.9$ at 4 weeks after 2 treatments $(n=26) ; 13.5 \pm 12.9$ at 6 weeks after 3 treatments $(n=22) ; 11.5 \pm 10.1$ at 8 weeks after 4 treatments $(\mathrm{n}=18)$; and $8.8 \pm 7.5$ at 10 weeks after 5 treatments $(n=13)$. Diode laser therapy in our study achieved a marked reduction of lesions by $13.9 \%, 28.2 \%, 43.3 \%$, $51.7 \%$, and $63.0 \%$, respectively (Figure 2). This improvement without statistically significance in Student's t-test showed a positive trend. Assessment of efficacy by the investigators showed that $96 \%$ of the patients had improved after 5 treatments, while $4 \%$ demonstrated an "unchanged" state (Table 1). Clinical pictures of two patients with marked improvement are shown in Figures 3-5. As seen in the figures, laser therapy with a 1450 -nm diode laser was effective for cysts and nodules as well as papules and pustules.

\section{Tolerability}

Only one patient discontinued treatment due to vesicle formation as an adverse effect, which resolved without pigmentation or scarring. All patients felt pain during the irradiation. Two patients felt moderate pain and 25 felt slight pain. This pain was transient and needed no treatment. No patients discontinued the study because of the pain from this modality.

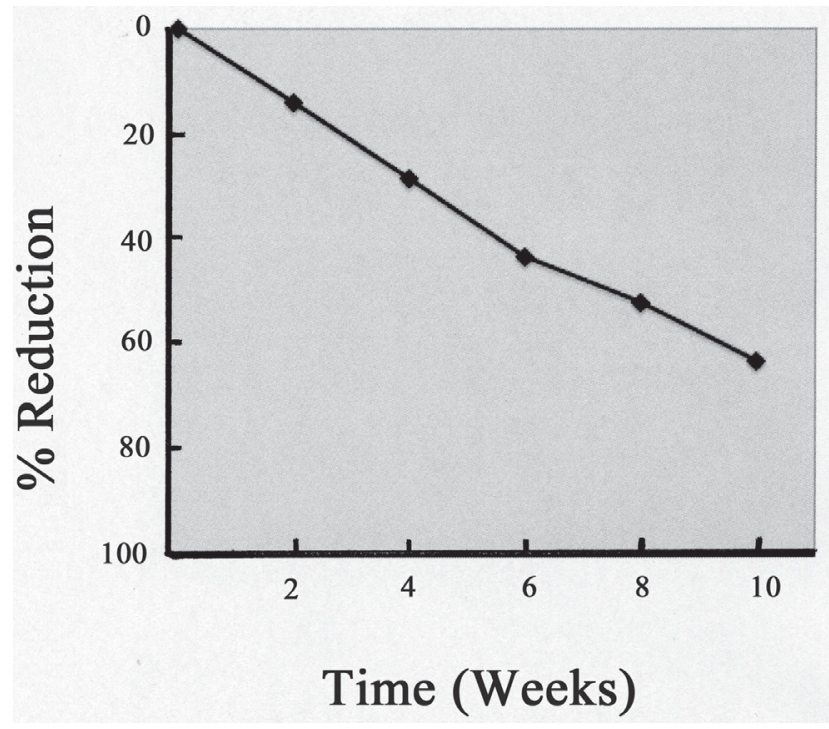

Figure 2 Counts of lesions (mean percentage reduction).
Table I Physician's overall ratings for the response of inflammatory acne

\begin{tabular}{ll}
\hline Rating & Number (\%) \\
\hline Worsened & $0(0 \%)$ \\
Unchanged & $1(4 \%)$ \\
Improved & $18(67 \%)$ \\
Markedly improved & $6(22 \%)$ \\
Resolved & $2(7 \%)$ \\
Total & $27(100 \%)$ \\
\hline
\end{tabular}

\section{Discussion}

The 1450-nm diode laser in our study demonstrated a marked effect on mild to moderate acne lesions and was well tolerated. The reduction of number of skin lesions was $51.7 \%$ at 8 weeks and $63.0 \%$ at 10 weeks. Ninety-six percent of patients showed improvement by week 10 although $4 \%$ of patients were unchanged. Only one patient showed vesicle formation following no pigmentation or scarring. Friedman and colleagues (2004) also reported minimal adverse effects from this diode laser therapy were transient erythema and edema. The 1450-nm diode laser may therefore be added to the panel of phototherapy used for acne treatment.

The 1450-nm laser with a dynamic cooling device has received Food and Drug Administration approval for the treatment of acne, atrophic acne scars, and fine wrinkles (Nouri and Ballard 2006). The light at $1450 \mathrm{~nm}$ in the infrared spectrum corresponds to the peak of absorption spectrum of water in the dermis, which indicates the target chromophore to be collagen and sebaceous gland in the mid dermis. Histopathological studies reveal that this laser causes thermal damage to the collagen (Paithankar et al 2002; Tanzi and Alster 2004) and sebaceous glands (Paithankar et al 2002). This laser is clinically effective for atrophic acne scar (Tanzi and Alster 2004) and sebaceous hyperplasia (No et al 2004). We hypothesize that the 1450-nm diode laser may remodel the collagen, form new collagen, and suppress the activity of sebaceous glands following an improvement of acne and acne scar tissue.

Blue light therapy has fewer effects on comedones than on inflammatory papules and pustules (Sigurdsson et al 1997; Papageorgiou et al 2000; Kawada, Aragane, Kameyama, et al 2002). Moreover, blue light emits the light of $415 \mathrm{~nm}$ that does not penetrate into the deep dermis, indicating blue light to be less effective for deeper acne lesions, eg, nodules and cysts. ALA-PDT is effective for acne vulgaris with significant side effects such as transient hyperpigmentation, superficial exfoliation, and crusting (Hongcharu et al 2000). The techniques of topical ALA- 
A

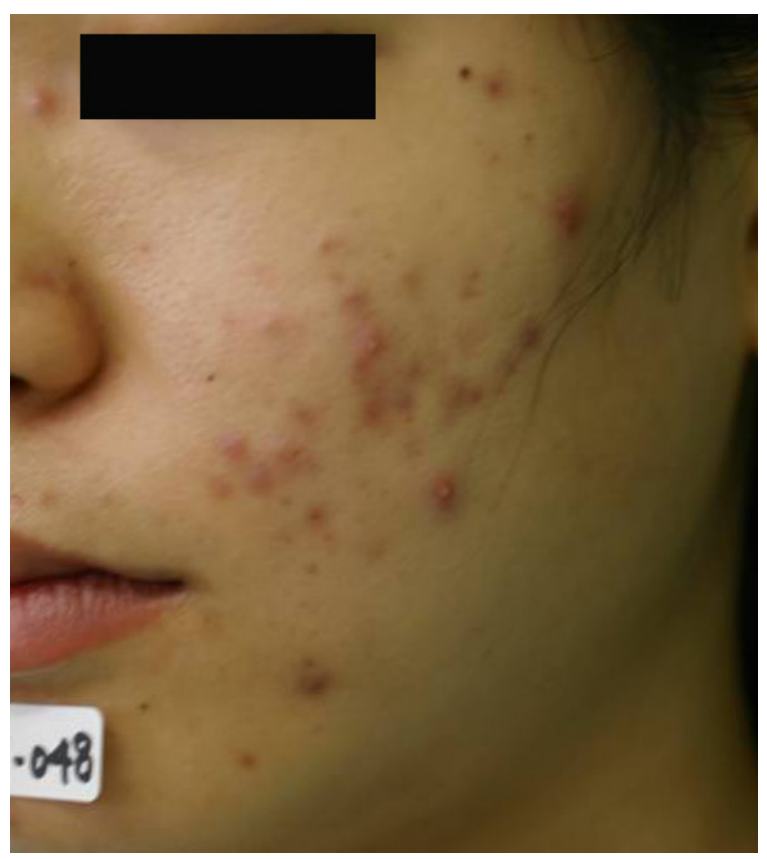

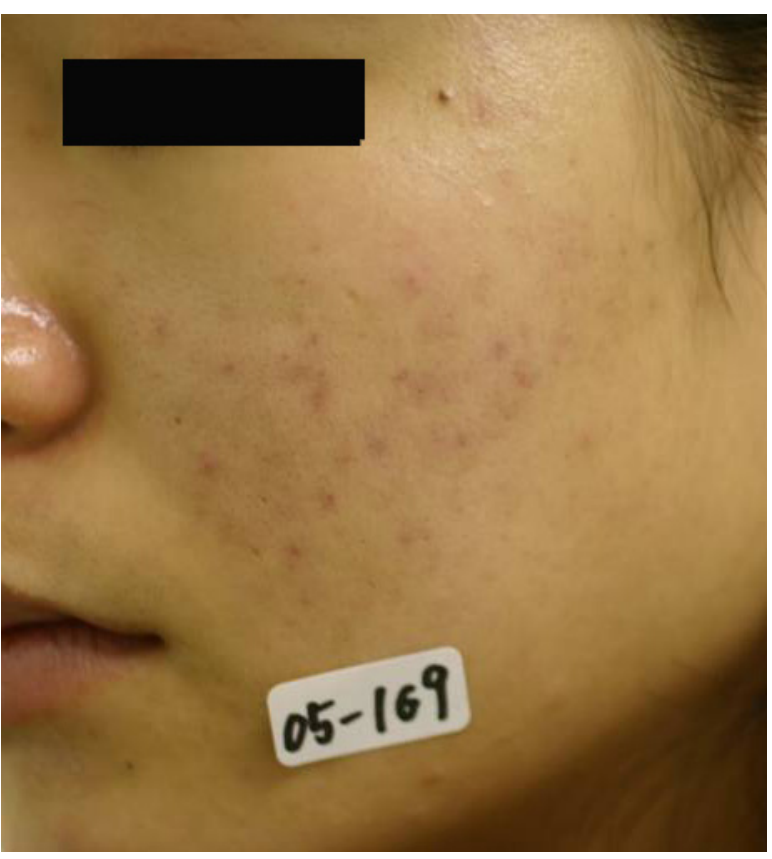

B

Figure 3 Case I (female, 20 years) with marked clinical improvement. A, before, and B, after the treatment.

photodynamic therapy for skin disorders have not been optimally established since inhomogenous distribution or lack of selective accumulation of ALA-derived PpIX (Peng et al 1997). Diode laser in our study was effective for nodules and cysts as well as papules and pustules with minimal adverse effects. Moreover, this laser therapy shows significant long-term clinical remission after treatment (Jih et al 2006). The 1450-nm diode laser may therefore have some advantages over blue light therapy and ALA-PDT.
In conclusion, our study demonstrated that $1450-\mathrm{nm}$ diode laser might be a treatment modality for mild to moderate acne.

\section{References}

Cunliffe WJ, Goulden V. 2000. Phototherapy and acne vulgaris. Br J Dermatol, 142:855-6.

Friedman PM, Jih MH, Kimyai-Asadi A, et al. 2004. Treatment of inflammatory facial acne vulgaris with the 1450-nm diode laser: a pilot study. Dermatol Surg, 30:147-51.
A

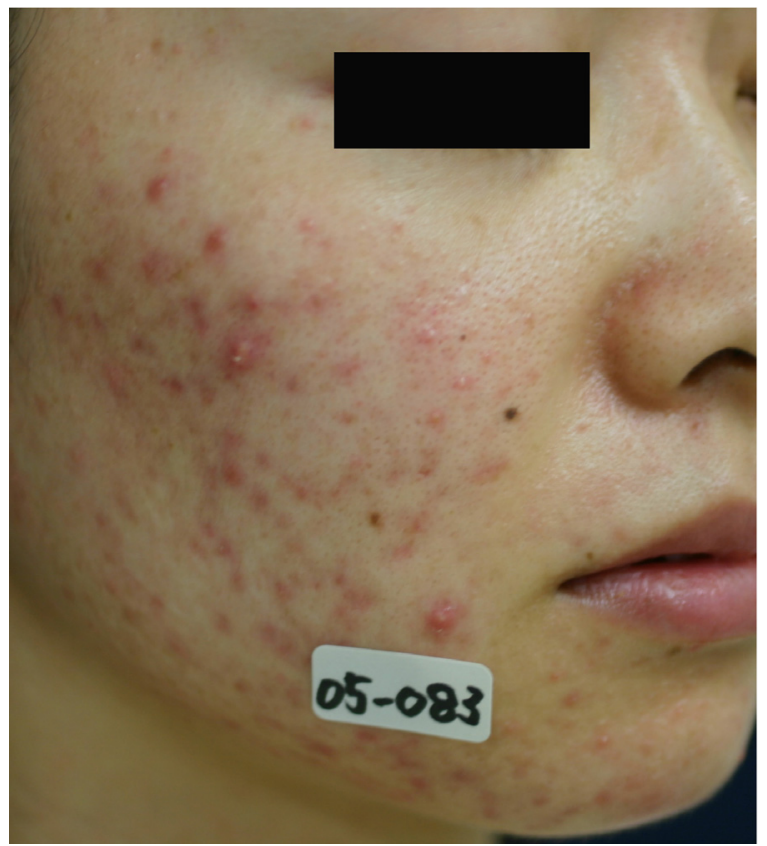

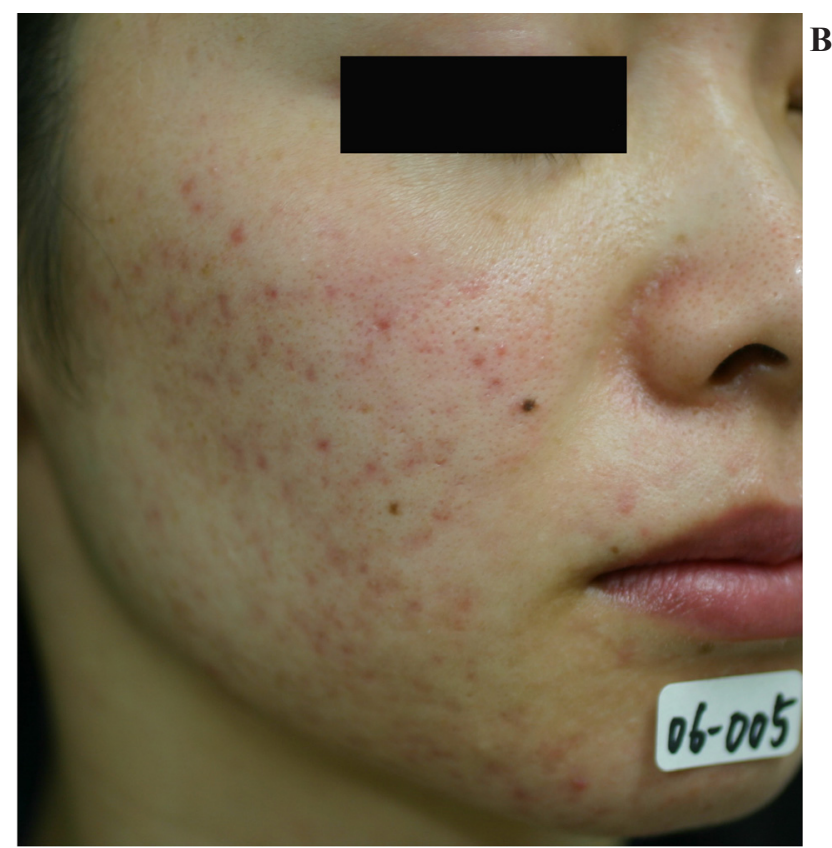

Figure 4 Case 2 (female, 28 years) with marked clinical improvement. A, before, and B, after the treatment. 

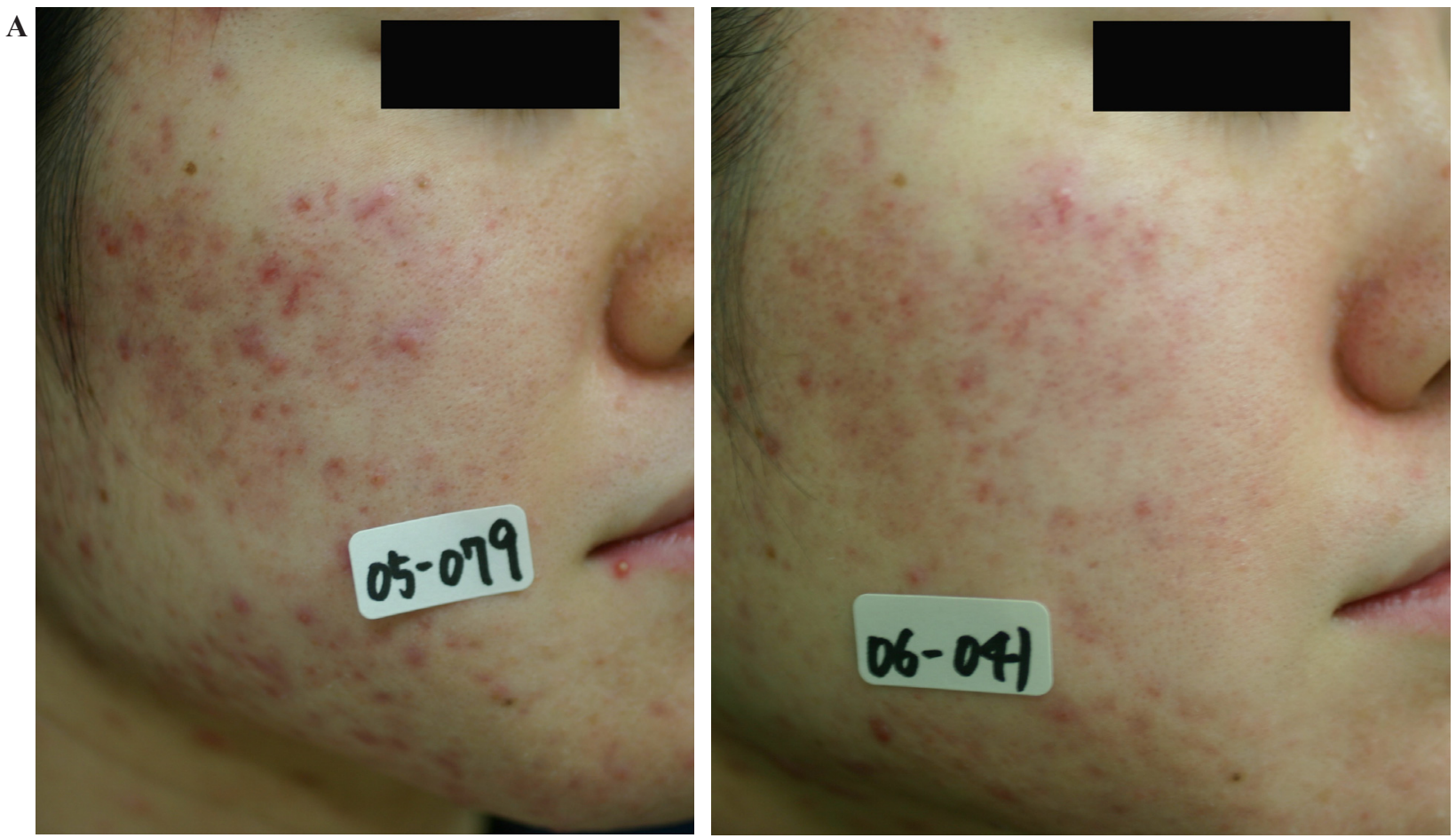

Figure 5 Case 3 (female, 21 years) with marked clinical improvement. A, before, and B, after the treatment.

Glass D, Boorman GC, Stables GI, et al. 1999. A placebo-controlled clinical trial to compare a gel containing a combination of isotretinoin $(0.05 \%)$ and erythromycin $(2 \%)$ with gels containing isotretinoin $(0.05 \%)$ or erythromycin $(2 \%)$ alone in the topical treatment of acne vulgaris. Dermatology, 199:242-7.

Hongcharu W, Taylor CR, Chang Y, et al. 2000. Topical ALAphotodynamic therapy for the treatment of acne vulgaris. $J$ Invest Dermatol, 115:183-92.

Jih MH, Friedman PM, Goldberg LH, et al. 2006. The 1450-nm diode laser for facial inflammatory acne vulgaris: dose-response and 12month follow-up study. J Am Acad Dermatol, 55:80-7.

Kawada A, Aragane Y, Tezuka T. 2002. Levofloxacin is effective for inflammatory acne and achieves high levels in the lesions: an open study. Dermatology, 204:301-2.

Kawada A, Aragane Y, Kameyama H, et al. 2002. Acne phototherapy with a high-intensity, enhanced, narrow-band, blue light source: an open study and in vitro investigation. J Dermatol Sci, 30:129-35.

Leyden JJ. 2003. A review of the use of combination therapies for the treatment of acne vulgaris. J Am Acad Dermatol, 49:S200-10.

Mariwalla K, Rohrer TE. 2005. Use of lasers and light-based therapies for treatment of acne vulgaris. Lasers Surg Med, 37:333-42.
No D, McClaren M, Chotzen V, et al. 2004. Sebaceous hyperplasia treated with a 1450-nm diode laser. Dermatol Surg, 30:382-4.

Nouri K, Ballard CJ. 2006. Laser therapy for acne. Clin Dermatol, 24:2632

Paithankar DY, Ross EV, Saleh BA, et al. 2002. Acne treatment with a $1,450 \mathrm{~nm}$ wavelength laser and cryogen spray cooling. Lasers Surg Med, 31:106-14.

Papageorgiou P, Katsambas A, Chu A. 2000. Phototherapy with blue (415 $\mathrm{nm})$ and red $(660 \mathrm{~nm})$ light in the treatment of acne vulgaris. $\mathrm{Br} J$ Dermatol, 142:973-8.

Peng Q, Warloe T, Berg K, et al. 1997. 5-aminolevulinic acid-based photodynamic therapy. Cancer, 79:2282-308.

Sigurdsson V, Knulst AC, van Weelden H. 1997. Phototherapy of acne vulgaris with visible light. Dermatology, 194:256-60.

Tanzi EL, Alster TS. 2004. Comparison of a 1450-nm diode laser and a 1320-nm Nd:YAG laser in the treatment of atrophic facial scars: a prospective clinical and histologic study. Dermatol Surg, 30:152-

Van De Kerkhof PC, Kleinpenning MM, de Jong EM et al. 2006. Current and future treatment options for acne. J Dermatol Treat, 17:198-204. 